\title{
Design, Analysis, and Control of a Low Power Joint for Walking Robots, by Phasic Activation of McKibben Muscles
}

\author{
Richard Q. van der Linde
}

\begin{abstract}
This paper describes the development of an efficient mechanical oscillator. It is capable of combining the energetic advantages of ballistic movement with cycle adjustability and active orbital control. Pneumatic artificial McKibben muscles are used as variable springs, from which the stiffness is varied in order to induce a limit cycle.
\end{abstract}

Index Terms - Limit cycle, McKibben muscles, phasic activation, variable passive stiffness.

\section{INTRODUCTION}

A UTONOMOUS robots are inevitably restricted to a limited amount of energy supply. This is even more the case for prosthetic and orthotic devices in rehabilitation technology, where weight is also an important design criterion. It would therefore be advantageous to design for minimal energy consumption and weight, especially when cyclic movements like walking are involved.

The first criterion can be fulfilled by choosing optimal movements and recycling energy as much as possible. From the viewpoint of energy consumption a typical optimal movement is one which utilizes the natural frequency of the system. This concept has been a leading topic in several robotic research projects: bipedal ballistic walking [8], [9], [16], self-excited vibratory actuators [19], joint compliance and trajectory selection [1], [3]. Very much related to the use of the natural frequency is energy recycling by spring like properties. Humans and mammals use this concept for running, hopping, and jumping [2], [17]. This principle is based on using the natural frequency of compliant structures. These concepts have been successfully utilized in many mobile robotic systems before, especially in hopping mono-, bi-, and quadrupeds [1], [3], [7], [18], [20], [21].

So using the natural frequency of the robot is shown to be a useful concept for energy efficient walking. However, the natural frequency of a system is determined by inherent system properties like: dimensions, mass distribution, collision behavior, and joint compliance. Constructing and controlling a desired movement with these parameters is not as easy as is the case with conventional servo mechanisms, which merely obey a calculated trajectory. The first method has its main point in lack of control parameters. The latter method shifts

Manuscript received August 13, 1997; revised March 23, 1999. This paper was recommended for publication by Associate Editor N. Ayache and Editor A. Goldenberg upon evaluation of the reviewers' comments.

The author is with the Delft University of Technology, Delft $2628 \mathrm{CD}$, The Netherlands (e-mail: R.Q.vanderLinde@wbmt.tudelft.nl).

Publisher Item Identifier S 1042-296X(99)05399-9. this shortcoming to a control problem, at the cost of high energy consumption and robust actuators. Since this trade off will most likely not lead to success in rehabilitation research and autonomous robotics, the first method is preferred.

Lack of control parameters implies no possibility to modify the limit cycle or to intervene when disturbances arise. This restriction can be eliminated by applying an adjustable system parameter. If mass distribution and dimensions are fixed, then joint compliance can be chosen to be adjustable. Active adjustment of joint stiffness offers the ability to:

1) add or withdraw energy to or from the system;

2) modify the natural frequency;

3) give the system a protection against high disturbance forces.

If energy storing properties are to be used also, then the compliance must be passive.

In comparison to active joint stiffness actuators, only little research on the area of passive joint stiffness actuators has been done. A good review is given by [27]. However, the goals of most of these researches were mainly focused on force control tasks [13], [23] or position control tasks [4], [14]. Passive springs have also been combined in series with (electric) displacement actuators [1], [3]. However, the eigenfrequency of the passive component is fixed, and a continuous torque must be delivered by the actuator. This paper will on the other hand merely focus on cyclic movements caused by active adjustment of passive stiffness. The acquired actively variable passive stiffness is realized by two opposite pneumatic artificial McKibben muscles.

First a model will be derived for a simple joint with two muscles. Amplitude and frequency, and the associated stability and energy consumption of a limit cycle as a function of pressure course parameters can then be obtained by return map analysis. Measurements of an experimental set-up showed that the model was able to predict the cyclic behavior rather well. The inverse solution is approximated by an analytic expression of the cyclic solutions map, which can serve as a discrete feedforward control. By adding feedback through modulation of the activation pulse, orbital stability can be improved significantly.

\section{The Phasic Actuated Variable Stiffness Joint}

In short it will be described how an experimental set-up was constructed. A nonlinear model of the complete experimental set-up is given. 


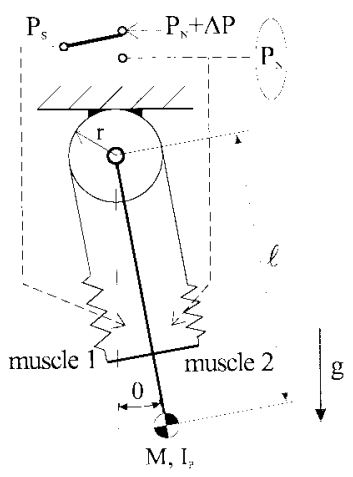

Fig. 1. Parameter conventions for the pendulum model. The muscles act as springs with a variable stiffness as a function of internal pressure. Therefore only the force-stretch relationship of the activation muscle varies in time.

\section{A. The Experimental Set-Up}

A simple pendulum model was chosen to be representative for swing leg behavior during walking. Two artificial pneumatic McKibben muscles were used as an agonist-antagonist couple (Fig. 1). A McKibben muscle is an actuator which converts pneumatic energy into mechanical energy by shortening [5], [10]-[12], [14], [28], [29]. It consists of an internal rubber tube covered with a nonelastic braiding. One end of the tube is closed, while the other end is the pneumatic input. When pressure is applied in the rubber tube the muscle will become stiffer. So adjusting the pressure in both muscles results in a joint stiffness change, comparable to cocontraction in biological systems.

A fixed point within the limit cycle can be chosen to trigger the activation of one of the muscles. This point is chosen to be the maximal amplitude of the joint angle, which is equal to a downwards triggered zero-crossing of the rate. Therefore a gyroscope was mounted at the end of the pendulum. The activation is obtained by raising the pressure by $\Delta P$ in the activation muscle. In the set-up a three-way valve was used to switch the pressure in muscle 1 between the nominal pressure and the activation pressure (Fig. 1). When an activation time $\Delta t$ has elapsed the supply pressure $P_{S}$ is set back to the nominal pressure $P_{N}$ again. The switching time of the valve is very small compared with the time scale of an activation pulse (Section III-A), and will therefore be neglected. Muscle 2 is kept on a constant pressure $P_{N}$.

In order to optimize energetic efficiency of the muscles dissipation was minimized by material choice. Different material combinations for braiding and tubing were tested by isotonic, quasi static stretching tests. Wall thickness of the tubing was a trade off between minimizing visco-elastic dissipation and maximal allowable muscle pressure. Due to a dense braiding the internal muscle pressure could be raised to a maximum of $3.5 \times 10^{5} \mathrm{~Pa}$ with only $0.2 \mathrm{~mm}$ wall thickness. With polyester braiding and latex tubing an efficiency of up to $94 \%$ could be achieved.

\section{B. Modeling the Experimental Set-Up}

The pneumatic muscle dynamics can be simplified by a lumped parameter model. The muscle volume changes as its length changes under a load. However, during contraction this change in volume will be small (maximal 5\% of the total muscle volume, at $18 \%$ muscle stretch). Therefore the muscle volume $\left(V_{M}\right)$ can be modeled as a constant accumulator, or capacitor $\left(C_{M}\right)$. The hose can be modeled as a resistor $(R)$. Modeling the activation muscle pressure $\left(P_{A M}\right)$ dynamics then results in a first order filter, given by the differential equation

$$
\dot{P}_{A M}(t)+\frac{P_{A M}(t)}{C_{M} R}=\frac{P_{S}(t)}{C_{M} R}
$$

where $C_{M}$ can be expressed by

$$
C_{M}=\frac{\Phi_{A M}}{\dot{P}_{A M}}=\frac{d m / d t}{d P_{A M} / d t}=\frac{d m}{d P_{A M}}
$$

where $\Phi_{A M}=$ the mass flow through the hose and $m=$ the gas mass. Using the ideal gas equation (2) can be rewritten as

$$
C_{M}=d m \cdot d\left(\frac{V_{M}}{m R_{\mathrm{GAS}} T}\right) \approx \frac{V_{M}}{R_{\mathrm{GAS}} T}
$$

where $R_{\mathrm{GAS}}=$ the specific gas constant and $T=$ gas temperature.

When dissipating friction forces are modeled by a viscous damping, the pendulums equation of motion according Fig. 1 can be written as

$$
\begin{aligned}
I_{P} \ddot{\theta}(t)= & -M g \ell \sin \theta(t)-F_{M 1}\left(\theta(t), P_{1}(t)\right) r \\
& +F_{M 2}\left(\theta(t), P_{2}\right) r-2 k r^{2} \dot{\theta}(t)
\end{aligned}
$$

where

$I_{P} \quad$ pendulum inertia around the joint;

$M \quad$ pendulum mass;

$g$ gravitational acceleration;

$\ell \quad$ distance from the joint to the center of gravity of the pendulum;

$F_{M n}$ force of muscle $n$ as a function of the pendulum angle $\theta$ and muscle pressure $P_{n}$;

$r \quad$ radius of the cylinder;

$k \quad$ constant of damping of one muscle.

Muscle 1 was chosen to be the activation muscle, so $P_{1}(t)=$ $P_{A M}(t)$, and muscle 2 was kept on a constant pressure $P_{N}$.

An approximation of the force-braiding angle relation of an ideal McKibben muscle has been derived by Chou and Hannaford (1996), rewriting gives

$$
F_{M}\left(\varepsilon, P^{\prime}\right)=\frac{b^{2} P^{\prime}}{4 \pi n^{2}}\left(3 \frac{L_{0}^{2}}{b^{2}}(1+\varepsilon)^{2}-1\right)
$$


TABLE I

VALUES OF THE SYSTEM PARAMETERS

\begin{tabular}{c|c|c|c|c|c} 
parameter & unit & value & parametcr & unit & valuc \\
\hline$\beta$ & - & 0.026 & $U_{i}$ & {$[\mathrm{~m}]$} & 0.01 \\
$C_{M}$ & {$\left[\mathrm{~kg} \cdot \mathrm{m}^{2} / \mathrm{N}\right]$} & $1.55 \cdot 10^{-10}$ & $s$ & {$\left[\mathrm{~m} / \mathrm{s}^{2}\right]$} & 0.81 \\
$R$ & {$[\mathrm{~N} \cdot \mathrm{s} / \mathrm{m}]$} & $2.80 \cdot 10^{8}$ & $\ell$ & {$[\mathrm{m}]$} & 0.273 \\
$R_{H i k}$ & {$[\mathrm{~J} / \mathrm{kg} \cdot \mathrm{K}]$} & 287.00 & $b$ & {$[\mathrm{~m}]$} & 0.1126 \\
$r$ & {$[\mathrm{~m}]$} & 0.018 & $m$ & {$[\mathrm{~kg}]$} & 0.309 \\
$V_{M}$ & {$\left[\mathrm{~m}^{3}\right]$} & $13.1 \cdot 10^{6}$ & $I_{-4}$ & {$[\mathrm{~m}]$} & 0.065 \\
$T$ & {$[\mathrm{~K}]$} & 293 & $I_{p}$ & {$\left[\mathrm{kgm}^{2}\right]$} & 0.0317 \\
$n$ & - & 1.83 & & & \\
\hline
\end{tabular}

There can be no stretch when there is no load, so the initial muscle length can be calculated as $L_{0}=(1 / 3) b \sqrt{3}$.

The strain of muscle $1\left(\varepsilon_{1}\right)$ and muscle $2\left(\varepsilon_{2}\right)$ can be expressed in $\theta$

$$
\varepsilon_{1}=\frac{U_{0}+r \theta}{L_{0}}, \quad \text { and } \quad \varepsilon_{2}=\frac{U_{0}-r \theta}{L_{0}}
$$

where $U_{0}=$ pre-elongation of the muscle.

$F_{M 1}$ and $F_{M 2}$ can now be obtained by substituting (6) in (5). Combining the result with (4) this yields an expression of the complete pendulum dynamics

$$
\begin{aligned}
\ddot{\theta}= & -\frac{M g \ell}{I_{P}} \sin \theta-\frac{2 k r^{2}}{I_{P}} \dot{\theta} \\
& -\frac{r b^{2} P_{A M}(t)}{4 \pi n^{2} I_{P}} \cdot\left(\frac{\left(U_{0}+r \theta\right)^{2}}{L_{0}^{2}}+\frac{2\left(U_{0}+r \theta\right)}{L_{0}}\right) \\
& +\frac{r b^{2} P_{N}}{4 \pi n^{2} I_{P}} \cdot\left(\frac{\left(U_{0}-r \theta\right)^{2}}{L_{0}^{2}}+\frac{2\left(U_{0}-r \theta\right)}{L_{0}}\right) .
\end{aligned}
$$

\section{Performance}

In this section, the outcome of the model will be discussed and validated. Return map analysis will be used to study the cycle properties.

\section{A. Experimental Versus Theoretical Results}

First the outcome of the model is compared with the results of the experimental set-up of which the values of the system parameters are given by Table I. The damping coefficient $\beta$ was determined by curve fitting of unactuated responses of the pendulum. This dimensionless damping ratio could be well approximated as a constant for the frequencies in the range of interest. Its relation to the constant of damping $k$ for one muscle according to (7) is given by

$$
k=\frac{\beta \omega_{B} I_{P}}{r^{2}}
$$

where $\omega_{B}=$ the natural frequency of the pendulum.

The time constant of the activation muscle can be found as $\tau_{A M}=R C_{M}=0.043 \mathrm{~s}$. Isotonic, quasi static stretch experiments were performed for different muscle pressures. With parameters according to Table I, (5) predicts muscle force with a mean error $2.2 \%$. Also it needs to be noted that $L_{0}$ remains constant in theory, but varied $3 \%$ in practice.

\section{B. Return Map Analysis}

In this paper, only cyclic motions are considered, since they form the essence of walking. Only frequency, amplitude, orbital stability, and energy consumption are cycle parameters of interest. These cycle properties must be quantified and expressed as a function of supply pressure course parameters: $P_{N}, \Delta P$, and $\Delta t$. Return map analysis is a useful tool in studying global dynamics of dynamic systems with periodic behavior [15], [24]. Fixed points are defined by the condition that their state in period $n$ equals their state in period $n+1$, thus can be obtained by finding the roots of the equation

$$
f(\boldsymbol{y})=\boldsymbol{P}\left(\boldsymbol{y}_{n}\right)-\boldsymbol{y}_{n+1}=\mathbf{0}
$$

where $\boldsymbol{f}(\boldsymbol{y})=$ a vector function and $\boldsymbol{P}\left(\boldsymbol{y}_{n}\right)=$ a mapping function which maps a state vector $\boldsymbol{y}\left(t_{n}\right)$ onto a state vector $\boldsymbol{y}\left(t_{n+1}\right)$.

A solution of this mapping equation can be found with a Newton Raphson method. For each combination of $\Delta P$ and $P_{N}$ (and choosing $\Delta t$ ) a matching cycle exists with certain cycle properties, plotted in Fig. 2(a)-(d) by contour lines. Within the working area of the muscles Fig. 2(a) gives lines of equal angle $\left(\Theta_{F}\right)$, and Fig. 2(b) gives lines of equal cycle period $\left(T_{F}\right)$. Fig. 2(c) gives lines of equal eigenvalues of the Jacobian of $\boldsymbol{P}$, which is a measure for the orbital stability [22]. The mapping function is a discrete function so the eigenvalues must lie within the unit circle to guarantee stability. All periodic solutions in Fig. 2(c) are slightly stable.

The power consumption $Q$ during one cycle is given by

$$
Q=\frac{1}{T} \oint_{\mathrm{CYCLE}} P_{A M} d V_{A M}
$$

where

$V_{A M}$ actuator muscle volume (no longer assumed to be constant!);

$P_{A M}$ actuator muscle pressure;

$T$ cycle period.

In Fig. 2(d) the power consumption is plotted for different settings of $\Delta P$ and $P_{N}$.

\section{Adjustability}

The return maps express an input parameter setting $(\Delta P$, $P_{N}$, and $\left.\Delta t\right)$ in periodic angle $\left(\Theta_{F}\right)$ and period $\left(T_{F}\right)$. However, the inverse relationship would be of great use too. The most simple way to find this inverse relationship is to approximate the return maps [Fig. 2(a) and (b)] by an analytical expression of which the inverse can be found easily. Such an approximation is given by

$$
\begin{aligned}
{\left[\begin{array}{c}
\hat{\Theta}_{F} \\
\hat{T}_{F}
\end{array}\right] } & =\left[\begin{array}{cc}
\frac{\partial \Theta}{\partial P_{N}} & \frac{\partial \Theta}{\partial \Delta P} \\
\frac{\partial T}{\partial P_{N}} & \frac{\partial T}{\partial \Delta P}
\end{array}\right] \cdot\left[\begin{array}{c}
P_{N} \\
\Delta P
\end{array}\right]+\left[\begin{array}{l}
K_{1} \\
K_{2}
\end{array}\right] \\
& =\nabla \boldsymbol{L} \cdot\left[\begin{array}{l}
P_{N} \\
\Delta P
\end{array}\right]+\boldsymbol{K}
\end{aligned}
$$

where

$\hat{\Theta}_{F} \quad$ estimation of the periodic pendulum angle in a fixed point [rad]; 


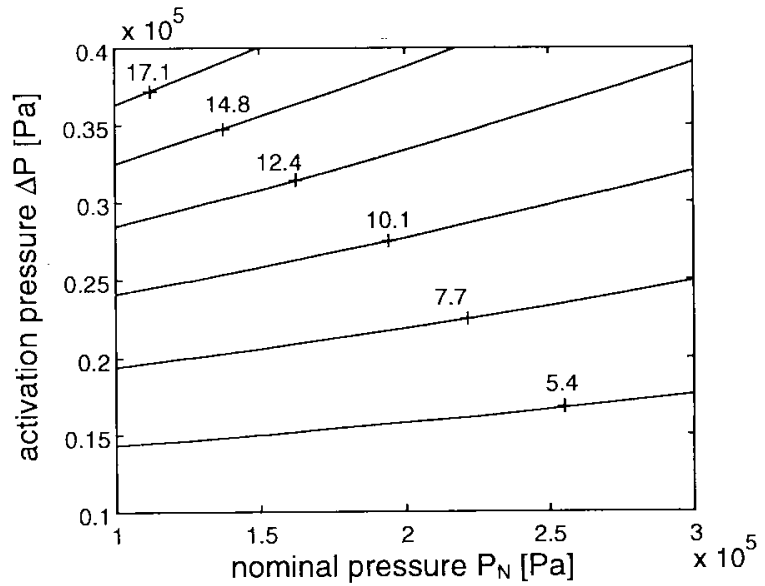

(a)

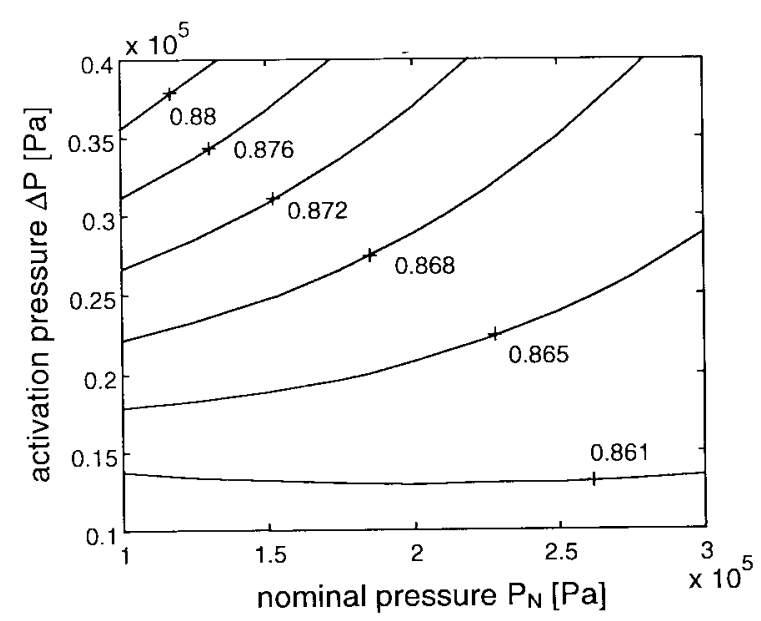

(c)

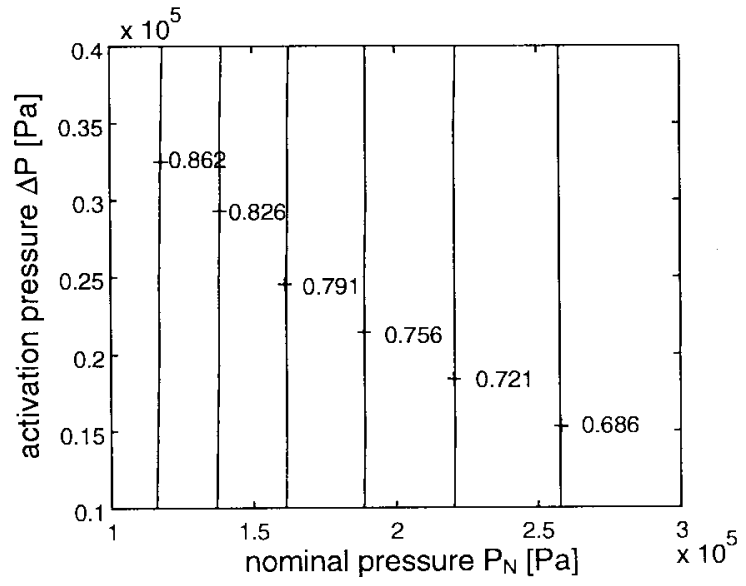

(b)

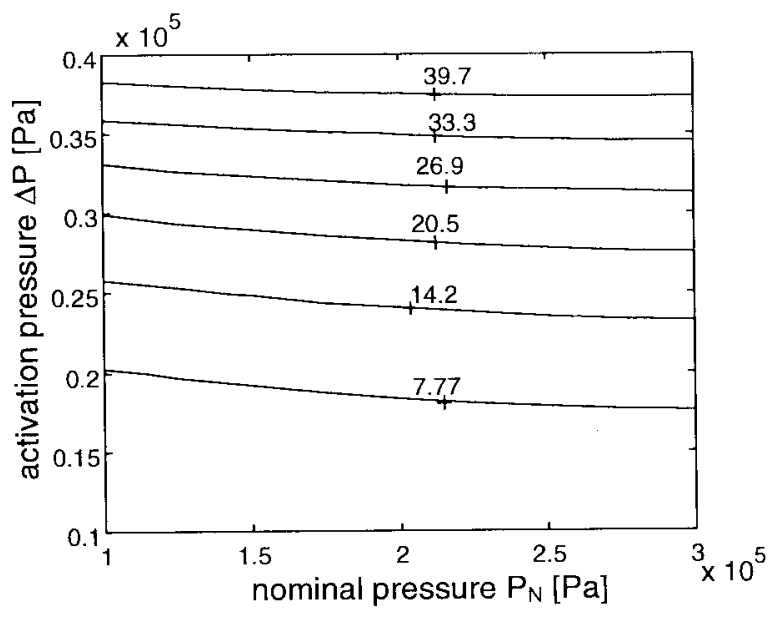

(d)

Fig. 2. (a)-(d) Results of a return map analysis for the nonlinear model. For each setting of $\Delta P$ and $P_{N}$ a fixed point is calculated. Each figure gives the lines of equal values of angle (a) $\left(\Theta_{F}\right) \mathrm{deg}$, (b) period $\left(T_{F}\right) \mathrm{s}$, (c) eigenvalue of the Jacobian, and (d) energy consumption $(Q)$ mW within each fixed point.

$\hat{T}_{F} \quad$ estimation of the periodic pendulum period in a fixed point [s];

$K_{1}, K_{2}$ constants.

$\boldsymbol{K}$ can be found by choosing an operational point in Fig. 2(a) and (b) for $\Delta P$ and $P_{N}$ for a specific $\Delta t$ and calculating $\Theta_{F}, T_{F}$, and $\nabla L$ by return map analysis. The working area of (11) after specifying an operational point covers the complete domain of pressure input parameters rather accurately, for Fig. 2(a) and (b) contain nearly straight, parallel lines. Equation (11) has a simple inverse, given by

$$
\left[\begin{array}{l}
P_{N} \\
\Delta P
\end{array}\right]=\nabla \boldsymbol{L}^{-1} \cdot\left\{\left[\begin{array}{l}
\hat{\Theta}_{F} \\
\hat{T}_{F}
\end{array}\right]-\boldsymbol{K}\right\} .
$$

The appropriate parameter settings for $P_{N}$ and $\Delta P$ in order to obtain a desired pendulum angle and period can now be found with (12).

\section{Controller Design}

The orbital stability was very low for each setting of $P_{N}$ and $\Delta P$ [a discrete pole around 0.9 , Fig. 2(c)], which implies slow convergence to the attractor after an external disturbance. This can be improved by adding a feedback, e.g., on the pulse width of the activation time $(\Delta t)$ of the activation muscle pressure (Fig. 3). Modulation of $\Delta t$ costs only very little extra gas consumption for the muscle volume remains nearly constant during contraction. If a small disturbance $\Delta \boldsymbol{y}_{n}$ occurs on a fixed point $\boldsymbol{y}_{F}$, then (9) can be approximated by

$$
\boldsymbol{y}_{n+1}+\Delta \boldsymbol{y}_{n+1} \approx \boldsymbol{P}\left(\boldsymbol{y}_{n}\right)+\nabla \boldsymbol{P}^{F} \Delta \boldsymbol{y}_{n}+\frac{\partial \boldsymbol{P}}{\partial \boldsymbol{X}} \Delta \boldsymbol{X}
$$

where

$$
\begin{array}{ll}
\boldsymbol{y}_{n} & \text { state space vector on time } n ; \\
\boldsymbol{P} & \text { mapping function; } \\
\nabla \boldsymbol{P}^{F} & \text { Jacobian of } \boldsymbol{P} \text { evaluated in fixed point } \boldsymbol{y}_{F} ; \\
\Delta \boldsymbol{y} & \text { deviation from the fixed point; } \\
\Delta \boldsymbol{X} & \text { control action. }
\end{array}
$$

A simple proportional discrete control law on activation time $\Delta t$ is chosen

$$
\Delta t=\Delta t_{N}+\Delta \boldsymbol{X}=\Delta t_{N}+G \Delta \boldsymbol{y}, \quad 0 \leq \Delta t \leq 1 / 2 T
$$

where $\Delta t_{N}=$ a nominal activation time and $\boldsymbol{G}=$ feedback gain. 


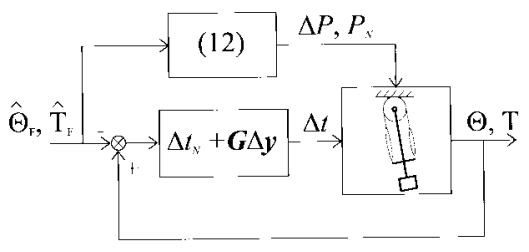

Fig. 3. Control scheme of the discrete controller, where the muscle activation $\Delta t$ is modulated by a proportional discrete feedback. A control action is summed with a nominal pulse width $\Delta t_{N}$. A feedforward according to (12) sets the right pressure settings. $\Delta t$ is limited in time by $0 \leq \Delta t \leq 1 / 2 T$.

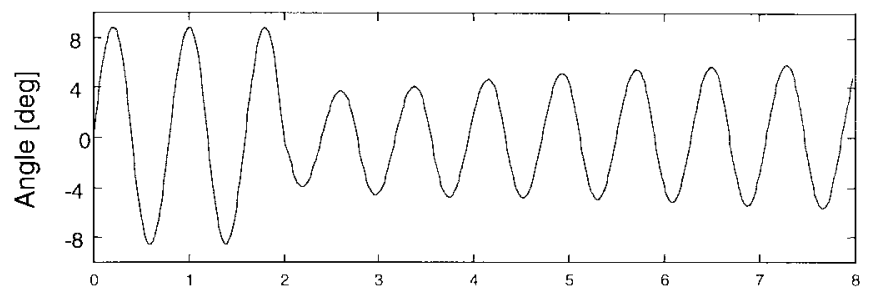

(a)

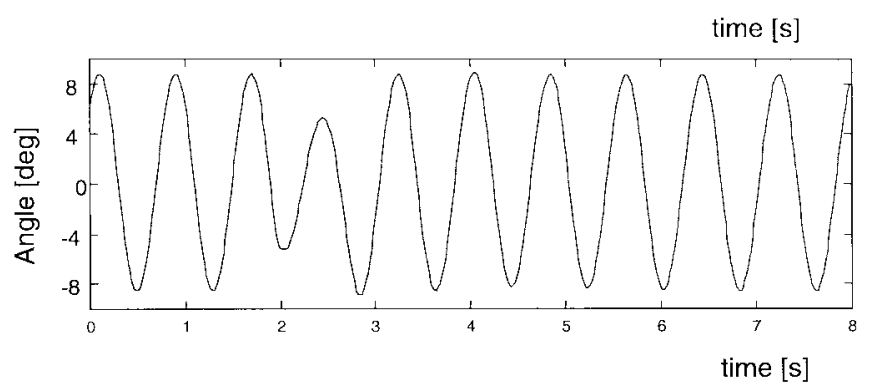

(b)

Fig. 4. Experimental results of the pendulum set-up for pressure settings $P_{N}=1.5 \cdot 10^{5} \mathrm{~Pa}, \Delta P=0.4 \cdot 10^{5} \mathrm{~Pa}, \Delta t_{N}=0.1 \mathrm{~s}$ : (a) without feedback control and (b) with optimal feedback control on $\Delta t$. At about time $t=2 \mathrm{~s}$ a perturbation is applied in both trials.

The boundaries on $\Delta t$ prevent the activation pressure to do negative work as the direction of motion changes. However, this mechanism could be used as a brake when $\Delta t$ becomes negative due to (14). Substituting (14) in (13) and canceling cyclic terms gives

$$
\Delta \boldsymbol{y}_{n+1}=\left(\nabla \boldsymbol{P}^{F}+\frac{\partial \boldsymbol{P}}{\partial \boldsymbol{X}} \boldsymbol{G}\right) \Delta \boldsymbol{y}_{n} .
$$

From (15) it can be seen that dead beat control is achieved for

$$
G=-\frac{\partial \boldsymbol{X}}{\partial \boldsymbol{P}} \nabla \boldsymbol{P}^{F}
$$

An experiment with the set-up was done for settings $P_{N}=$ $1.5 \cdot 10^{5} \mathrm{~Pa}, \Delta P=0.4 \cdot 10^{5} \mathrm{~Pa}, \Delta t=0.1 \mathrm{~s}$ and during a steady oscillation a perturbation was applied [Fig. 4(a)]. Next we numerically calculated the fixed point with (1), (7), and (9) for these settings. Then $\left(\partial \Theta_{F} / \partial \Delta t\right)=0.256$ and $\nabla \boldsymbol{P}^{F}=0.866$ were calculated within the fixed point. With (16) we find a gain: $\boldsymbol{G}=-3.38$. This controller setting was applied in the set-up and again a perturbation was applied during steady oscillation [Fig. 4(b)]. Within one cycle the pendulum had returned to its orbit. This result clearly shows the effectiveness of the introduced control action.

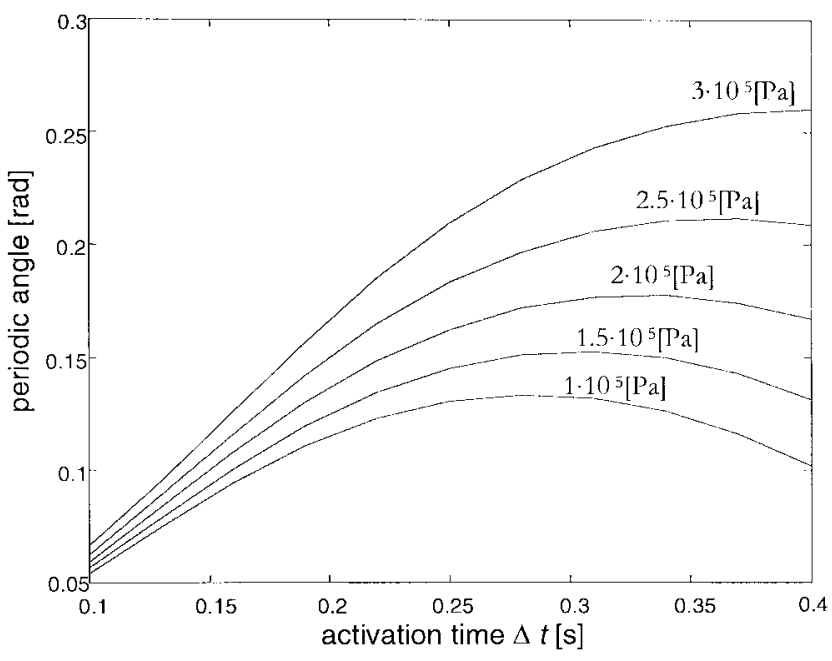

Fig. 5. The effect of the activation time $\Delta t$ on the periodic angle $\Theta_{F}$ as a function of pressure parameter $P_{N}$.

However, according to (16) an optimal gain is a function of the effect of the applied control action (here: the activation time $\Delta t$ ) on the return map (here: periodic angle), and the Jacobian. Fig. 2(c) shows that the Jacobian changes only slightly as a function of pressure settings $\Delta P$ and $P_{N}$. In Fig. $5 \Theta_{F}$ is plotted against $\Delta t$ as a function of $P_{N}$. Clearly $\left(\partial \Theta_{F} / \partial \Delta t\right)$ is not a constant in the working area of $P_{N}$, thus the value of an optimal gain is cycle dependent.

\section{CONCLUSION}

In this paper a variable passive stiffness joint with phasic activation is presented. By utilizing the spring-like properties of energetically optimized pneumatic artificial muscles a mechanical oscillator with low power consumption can be constructed. It is capable of combining passive motions with active actuation and control.

A pendulum model was chosen to be representative for swing leg behavior of walking robots. Two antagonistic McKibben muscles realize a joint stiffness, approximately proportional to the internal muscle pressure. Limit cycles are induced by a phasic change of the stiffness of one of the muscles. With this, cycle properties like amplitude and frequency are individually adjustable, and for each cycle a dead beat proportional discrete control setting can be calculated. An experimental pendulum set-up was constructed according to this principle. Its energy consumption lies within the range of 5-50 $\mathrm{mW}$, depending on amplitude and frequency. A nonlinear model was derived of which the overall behavior matched the observations rather accurately.

Eventually this joint is to be implemented in the hips of a currently existing prototype biped [25]. This implies different dynamic conditions in which this joint will have to operate. Collision behavior will be present due to foot contact at the end of the swing phase. The swing leg will then become stance leg and visa versa. However, once a stable walking cycle is constructed [26], the orbit of a swing leg will be known. This orbit will be different from the isolated pendulum studied in 
this paper. Still, this work can serve as a basis for, e.g., foot placement by swing leg control.

\section{REFERENCES}

[1] M. Ahmadi and M. Buehler, "Stable control of a simulated onelegged running robot with hip and leg compliance," IEEE Trans. Robot. Automat., vol. 13, pp. 96-104, Feb. 1997.

[2] R. M. Alexander, "Three uses for springs in legged locomotion," Int. J. Robot. Res., vol. 9, no. 2, pp. 53-61, 1990.

[3] M. D. Berkemeier and K. V. Desai, "Design of a robot leg with elastic energy storage, comparison to biology, and preliminary experimental results," in Proc. IEEE Int. Conf. Robot. Automat., Minneapolis, MN, Apr. 22-28, 1996, pt. I, pp. 213-218.

[4] D. G. Caldwell, G. A. Medrano-Cerda, and M. Goodwin, "Control of pneumatic muscle actuators," IEEE Trans. Contr. Syst., pp. 40-48, Feb. 1995.

[5] D. G. Caldwell, G. A. Medrano-Cerda, and C. J. Bowler, "Investigation of bipedal robot locomotion using pneumatic muscle actuators," in Proc. IEEE ICRA'97, Albuquerque, NM, Apr. 1997, pp. 799-804.

[6] C.-P. Chou and B. Hannaford, "Measurement and modeling of McKibben pneumatic artificial muscles," IEEE Trans. Robot. Automat., vol. 12, pp. 90-102, Feb. 1996.

[7] S. Dhandapani and M. M. Ogot, "Modeling of a leg system to illustrate the feasibility of energy recovery in walking machines," $A S M E A d v$. Design Automat., vol. 69, no. 2, pp. 429-436, 1994.

[8] B. Espiau and A. Goswami, "Compass gait revisited," in Proc. IFAC Symp. Robot Contr., Sept. 1994, pp. 830-846.

[9] M. Garcia, A. Chatterjee, A. Ruina, and M. Coleman, "The simplest walking model: Stability, complexity, and scaling," ASME J. Biomed. Eng., Apr. 16, 1997.

[10] J. J. Grodski and G. B. Immega, "Myoelectric control of compliance on a ROMAC protoarm," in Proc. Int. Symp. Teleop. Contr., July 1988, pp. 297-308.

[11] B. Hannaford, J. M. Winters, C.-P. Chou, and P.-H. Marbot, "The anthroform biorobotic arm: A system for the study of spinal circuits," Ann. Biomed. Eng., vol. 23, pp. 399-408, 1995.

[12] "Actuator properties and movement control: Biological and technological models," Multiple Muscle Systems: Biomechanics and Movement Organization, J. M. Winters and S. L.-Y. Woo, Eds. New York: Springer-Verlag, 1990, ch. 7, pp. 101-120.

[13] M. Hashimoto and Y. Imamura, "An instrumented compliant wrist using a parallel mechanism," in Proc. ASME Japan/USA Symp. Flexible Automat., 1992, vol. 1, pp. 741-744.

[14] K. Inoue, "Rubbertuators and applications for robotics," in Robotics Research: The 4th International Symposium, R. Bolles and B. Roth Eds. Cambridge, MA: MIT Press, 1988, pp. 57-63.

[15] D. E. Koditschek and M. Bühler, "Analysis of a simplified hopping robot," Int. J. Robot Res., vol. 10, no. 6, pp. 587-605, 1991.

[16] T. McGeer, "Passive dynamic biped catalogue," in Proc. 2nd Int. Symp. of Experimental Robotics. New York: Springer-Verlag, June 1991, pp. 465-490.
[17] T. A. McMahon, "Spring-like properties of muscles and reflexes in running," in Multiple Muscle Systems: Biomechanics and Movement Organization, J. M. Winters and S. L.-Y. Woo, Eds. New York: Springer-Verlag, 1990, pp. 578-590

[18] G. Mennitto and M. Buehler, "Experimental validation of compliance models for LADD transmission kinematics," in Proc. IEEE Conf. Intell. Syst. Robots, Pittsburgh, PA, Aug. 1995, pp. 385-390.

[19] K. Ono and K. Okada, "Self-excited vibratory actuator-3rd Report: Biped walking mechanism by self-excitation," Trans. Jpn. Soc. Mech. Eng., vol. 60, pp. 3711-3718, Nov. 1994, in Japanese.

[20] H. Rad, P. Gregorio, and M. Buehler, "Design modeling and control of a hopping robot," in Proc. IEEE/RSJ Conf. Intell. Syst. Robots, Yokohama, Japan, July 1993, pp. 1778-1785.

[21] M. H. Raibert, Legged Robots That Balance. Cambridge, MA: MIT Press, 1986.

[22] R. Seydel, Practical Bifurcation and Stability Analysis, 2nd ed. New York: Springer-Verlag, 1986.

[23] S. Sugano, S. Tsuto, and I. Kato, "Force control of the robot finger joint equipped with mechanical compliance adjuster," in Proc. IEEE/RSJ Int. Conf. Int. Robot. Syst., Raleigh, NC, July 7-10, 1992, pp. 2005-2013.

[24] A. F. Vakakis, J. W. Burdick, and T. K. Caughey, "An 'interesting' strange attractor in the dynamics of a hopping robot," Int. J. Robot. Res., vol. 10, no. 6, pp. 606-618, Dec. 1991.

[25] R. Q. Van der Linde and D. H. Plettenburg, "Synthesis of a simple ballistic walking movement with push-off," Rehabilitation R\&D Progr. Rep., vol. 34, pp. 44-45, May 1997.

[26] R. Q. Van der Linde, "Active leg compliance for passive walking," in Proc. IEEE Int. Conf. Robot. Automat. 1998, Leuven, Belgium, May 16-20, 1998, pp. 2339-2345.

[27] M. M. Williamson, "Series elastic actuators," A.I. Tech. Rep. 1524, Ph.D. dissertation, Mass. Inst. Technol., Cambridge, MA, 1995.

[28] J. M. Winters and E. S. Sagranichiny, "Why braided pneumatic actuator in rehabilitation robotics? Principles, properties, \& suggested applications," in Proc. 4th Int. Conf. Rehab. Rob., Wilmington, NC, June 1994, pp. $201-208$

[29] J. M. Winters and J. D. Peles, "Neck muscle activity and 3-D head kinematics during quasistatic and dynamic tracking movements," in Multiple Muscle Systems: Biomechanics and Movement Organization, J. M. Winters and S. L.-Y. Woo, Eds. New York: Springer-Verlag, 1990, ch. 28, pp. 461-480.

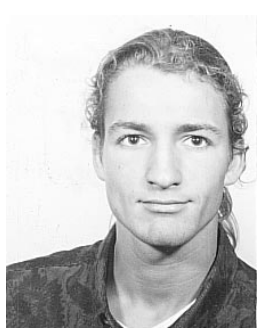

Richard Q. van der Linde was born in 1970. He received the M.Sc. degree in mechanical engineering from the Delft University of Technology, The Netherlands, in 1995, where he is currently pursuing the Ph.D. degree at the Man-Machine Systems and Control Group.

His current research interests are biologically inspired robots, biomechanics, limit cycles and control, and pneumatic artificial muscles. 